\title{
Intracellular and Extracellular Lipopolysaccharide Signaling in Sepsis: Avenues for Novel Therapeutic Strategies
}

\author{
Ramy S. Gabarin ${ }^{a} \quad$ Manshu Li $i^{a, b} \quad$ Paige A. Zimmel ${ }^{a} \quad J o h n$ C. Marshalla, c \\ Yimin Li ${ }^{a}$, b Haibo Zhanga, b, c, d, e \\ ${ }^{a}$ Keenan Research Center for Biomedical Science of Unity Health Toronto, Toronto, ON, Canada; ${ }^{\text {b}}$ The State Key \\ Laboratory of Respiratory Disease, and the 1st Affiliated Hospital of Guangzhou Medical University, Guangzhou, \\ China; Interdepartmental Division of Critical Care Medicine, University of Toronto, Toronto, ON, Canada; \\ ${ }^{\mathrm{d} D e p a r t m e n t}$ of Anesthesia, University of Toronto, Toronto, ON, Canada; eDepartment of Physiology, University of \\ Toronto, Toronto, ON, Canada
}

\section{Keywords}

Sepsis · Lipopolysaccharide · Intracellular signaling ·

Pyroptosis · Therapy

\begin{abstract}
Sepsis is defined as organ dysfunction due to a dysregulated systemic host response to infection. During gram-negative bacterial infection and other acute illness such as absorption from the gut infection, lipopolysaccharide (LPS) is a major mediator in sepsis. LPS is able to trigger inflammation through both intracellular and extracellular pathways. Classical interactions between LPS and host cells first involve LPS binding to LPS binding protein (LBP), a carrier. The LPS-LBP complex then binds to a receptor complex including the CD14, MD2, and toll-like receptor 4 (TLR4) proteins, initiating a signal cascade which triggers the secretion of pro-inflammatory cytokines. However, it has been established that LPS is also internalized by macrophages and endothelial cells through TLR4-independent pathways. Once internalized, LPS is able to bind to the cytosolic receptors caspases- $4 / 5$ in humans and the homologous caspase- 11 in mice. Bound caspases- $4 / 5$ oligomerize and trigger the assembly of the nucleotide-binding domain, leucine-rich-containing family,
\end{abstract}

pyrin domain-containing-3 inflammasome followed by the activation of inflammatory caspase- 1 resulting in subsequent release of interleukin- $1 \beta$. Caspases- $4 / 5$ also activate the perforin gasdermin $D$ and purinergic receptor $\mathrm{P} 2 \mathrm{X} 7$, inducing cell lysis and pyroptosis. Pyroptosis is a notable source of inflammation and damage to the lung endothelial barrier during sepsis. Thus, inhibition of caspases- $4 / 5 / 1$ or downstream effectors to block intracellular LPS signaling may be a promising therapeutic approach in adjunction with neutralizing extracellular LPS for treatment of sepsis.

(C) 2021 The Author(s).

Published by S. Karger AG, Basel

\section{Introduction}

Sepsis, defined as organ dysfunction due to a dysregulated host response to an infection, has a mortality rate near $40 \%$ [1]. Lipopolysaccharide (LPS) is the most common microbial mediator in sepsis and septic shock [2]. LPS is a major component of the outer membrane of gram-negative bacteria and a pathogen-associated molecular pattern which is recognized by the pattern recognition receptor toll-like receptor 4 (TLR4) $[3,4]$. Once host cells recognize LPS, they release pro-inflammatory
C 2021 The Author(s).

Published by S. Karger AG, Basel

This is an Open Access article licensed under the Creative Commons Attribution-NonCommercial-4.0 International License (CC BY-NC) (http://www.karger.com/Services/OpenAccessLicense), applicable to the online version of the article only. Usage and distribution for commercial purposes requires written permission.
Correspondence to:

Yimin Li, dryiminli@vip.163.com

Haibo Zhang, haibo.zhang@unityhealth.to 
cytokines including tumor necrosis factor- $\alpha$, interleukins (ILs) $[5,6]$. If an imbalance occurs between pro- and anti-inflammatory mediators, the inflammatory responses are dysregulated leading to the development of sepsis. It should be noted that sepsis can also be caused by grampositive bacterial infections and in some cases by fungi or parasites. In such cases, LPS would not play a role in pathogenesis [2].

While the relationship between extracellular LPS and TLR4 has been well documented, pharmacological interventions to neutralize circulating LPS or to block cell surface TLR4 receptors have failed in clinical trials [7]. One possible explanation for these failures is unabated LPS internalization in diverse cell types. Human lung epithelial cells, endothelial cells (ECs), cardiomyocytes, and macrophages can all internalize LPS in vitro [8-11]. LPS has been shown to enter the cytoplasm of macrophages and induce inside-out pro-inflammatory signaling independent of TLR4 [11-14]. Once internalized, LPS binds to the inflammatory caspases- $4 / 5$ in humans and their ortholog caspase-11 in mice, resulting in the activation of the nucleotide-binding domain, leucine-rich-containing family, pyrin domain-containing-3 (NLRP3) and "noncanonical" inflammasomes, leading to pyroptosis, an inflammatory form of cell death. Pyroptosis of both macrophages and ECs is a contributor to inflammation and endothelium dysfunction in sepsis. Ongoing research into inhibitors of intracellular LPS signaling has shown potential in mouse sepsis models. In this review paper, we will focus discussion on how intracellular LPS is a relevant, novel therapeutic target in sepsis-associated with gram-negative bacterial infection.

\section{Intracellular LPS Signaling}

Recent studies have established the biological importance of intracellular LPS in immune response [15]. Numerous mechanisms have been proposed for LPS internalization including endocytosis of outer membrane vesicles (OMVs), endotoxin delivery protein hepatocyte-released high-mobility group (HMGB1), as well as CD14/TLR4-dependent and independent pathways [16-18]. Once inside the cytosol, LPS then acts on intracellular receptors, most notably the murine caspase- 11 and the human caspases- $4 / 5$ [12-14]. Upon binding with LPS, these caspases oligomerize and activate a number of cytotoxic effectors. These include gasdermin D (GSDMD), caspase-1, and purinergic receptor P2X7. Upon effector activation, the cell begins to secrete IL- $1 \alpha / \beta$, IL-6, IL-18, and undergo pyroptosis, an in- flammatory form of cell death in macrophages, ECs, and epithelial cells $[9,12,19]$. Pyroptosis of both macrophages and ECs increases levels of inflammation and causes the destruction of the lung endothelial barrier, contributing to mortality in sepsis $[9,20]$.

\section{Possible Mechanisms for LPS Internalization}

A number of LPS internalization pathways are suggested in the literature, including OMVs, delivery via box 1 HMGB1, and direct transport of LPS-LBP complexes. Evidence shows that OMVs produced by gram-negative bacteria can deliver LPS to the cytosol of macrophages. These vesicles interact well with cellular membranes and are transported more readily across cell membranes than free-form LPS [16]. OMVs are secreted by bacteria in a programmable fashion under stressful conditions, such as in a host environment, allowing bacteria to secrete active virulence factors which facilitates pathogenesis [21]. OMVs are internalized by host cells through endocytosis and inserted into endosomes. LPS is then released into the cytosol from these early endosomes (shown in Fig. 1a). It should be noted that Vanaja et al. [16] showed that LPS internalization can occur regardless of bacterial phagocytosis. Macrophages treated with cytochalasin D, which inhibits actin polymerization and thus phagocytosis, had equal levels of cytosolic LPS as a control [16].

HMGB1 has also been suggested as a means of LPS internalization. In both macrophages and ECs, HMGB1 appears to bind extracellular LPS, forming HMGB1-LPS complexes which are internalized into endo-lysosomes via the receptor for advanced glycation end-products. HMGB1-LPS complexes are then delivered to acidic lysosomes. HMGB1 permeabilizes lysosome membranes, inducing release of LPS into the cytosol and subsequent caspase- 11 activation (shown in Fig. 1b). It was also found that cells must be primed for this pathway with doses of extracellular LPS. Additionally, oxidized HMGB1 can interact directly with the TLR4/MD2 complex as a priming signal for subsequent NLRP3 inflammasome activation. Notably, hepatocyte-specific $\mathrm{Hmgbl}$ gene deletions fail to protect mice from single high LPS doses, as demonstrated by Huebener et al. [22]. Contrastingly, Deng et al. [17] found that these deletions are protective in a double-hit endotoxemia model with an initial low, priming LPS dose, suggesting high LPS concentrations may bypass the HMGB1 pathway [22].

LPS binds to LBP in the serum, then may be internalized directly as a means of plasma clearance of LPS (shown in Fig. 1c). LPS uptake in CD14 deficient monocytes and ECs may rely on uptake of LPS-LBP complexes via scav- 
Fig. 1. Overview of LPS internalization pathways. a An OMV is internalized via clathrin-mediated endocytosis. LPSs are then able to diffuse from early endosomes into the cytosol. b Hepatocytes release HMGB1 into circulation which form complexes with LPS. These HMGB1-LPS complexes are internalized via RAGE-mediated endocytosis then transported into lysosomes. HMGB1 permeabilizes lysosomal membranes, allowing LPS to access the cytosol. c Evidence suggests LPS-LBP complexes are able to enter the cytosol through unknown pathways. Diagram created with Biorender.com. LPS, lipopolysaccharide; LBP, LPS binding protein; HMGB1, hepatocyte-released high-mobility group; RAGE, receptor for advanced glycation end-products; OMV, outer membrane vesicle.

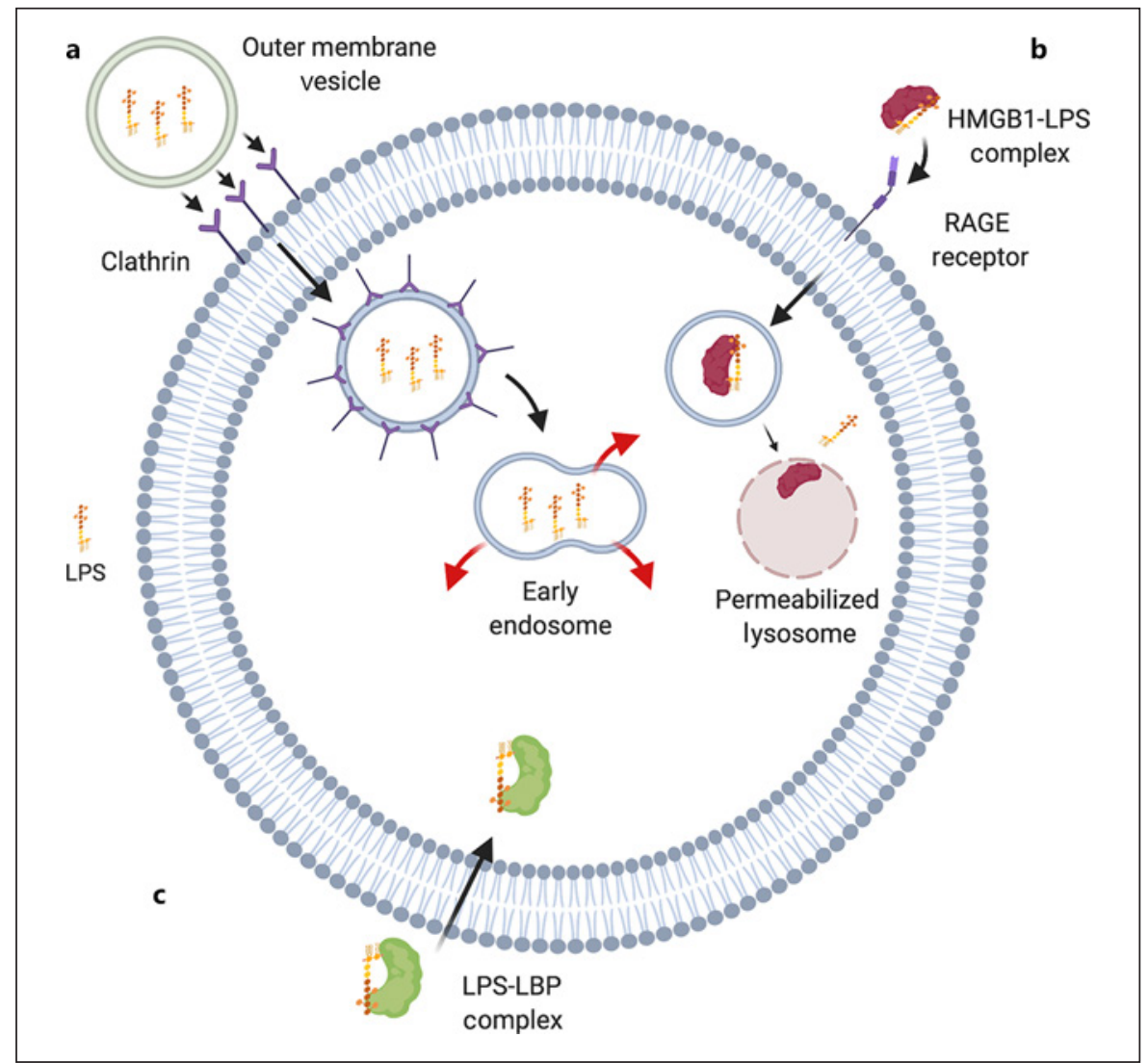

enger receptors [23, 24]. Kopp et al. [11] demonstrated that LBP colocalizes with LPS in intracellular compartments and elicits cytokine release in TLR4 and CD14 deficient monocytes. This suggests LPS-LBP complexes can cross cell membranes independent of the CD14/TLR4 complex. Thus, LBP might function in internalizing LPS as well as being an extracellular carrier. LBP has been shown to interact with lipids and even intercalate into phospholipid bilayers, potentially allowing it to be internalized directly [11]. Holistically, more research is needed to determine which of these internalization pathways are the most significant contributors to LPS signaling or if there are other potential routes of LPS uptake.

\section{Intracellular LPS Receptors and Effectors}

Caspase-11/4/5 Bind Cytoplasmic LPS

Recent studies have described the activation of both canonical and "noncanonical" inflammasomes in response to cytoplasmic LPS, whereby LPS binds to the murine caspase- 11 or the human caspases- $4 / 5$, inducing cytokine secretion and pyroptosis in macrophages [13]. When LPS is carried into the cytoplasm via cholera toxin
$\mathrm{B}$, murine caspase- 11 is activated, resulting in subsequent pyroptosis [14, 25]. Shi et al. [12] used LPS electroporation to artificially transport LPS and found that it binds to human caspase- 4 , then induces inflammation and pyroptosis. Artificial transfection of LPS is not necessarily an accurate reflection of sepsis in patients. However, such studies have provided other evidence suggesting caspases are important to the LPS response. Shi et al. [12] found that knockout of the CASP4 gene with either siRNA or CRISPR/Cas9 blocked LPS-induced cytotoxicity.

Caspases-11/4/5 are structurally similar to apoptotic initiator caspases, and all contain an amino-terminal caspase activation and recruitment domain [14]. Caspase-11/4/5 bind to the lipid A moiety of LPS with the lysine residues on their caspase activation and recruitment domain domains then oligomerize (shown in Fig. 2a, b) $[12,14,25]$. Caspase-11/4/5 oligomerization is followed by the assembly of the NLRP3 inflammasome, activation of caspase-1, cleavage of GSDMD, and subsequent pyroptosis.

Hagar et al. [25] and Kayagaki et al. [13] have shown that caspase-11 activation and a subsequent endotoxic 


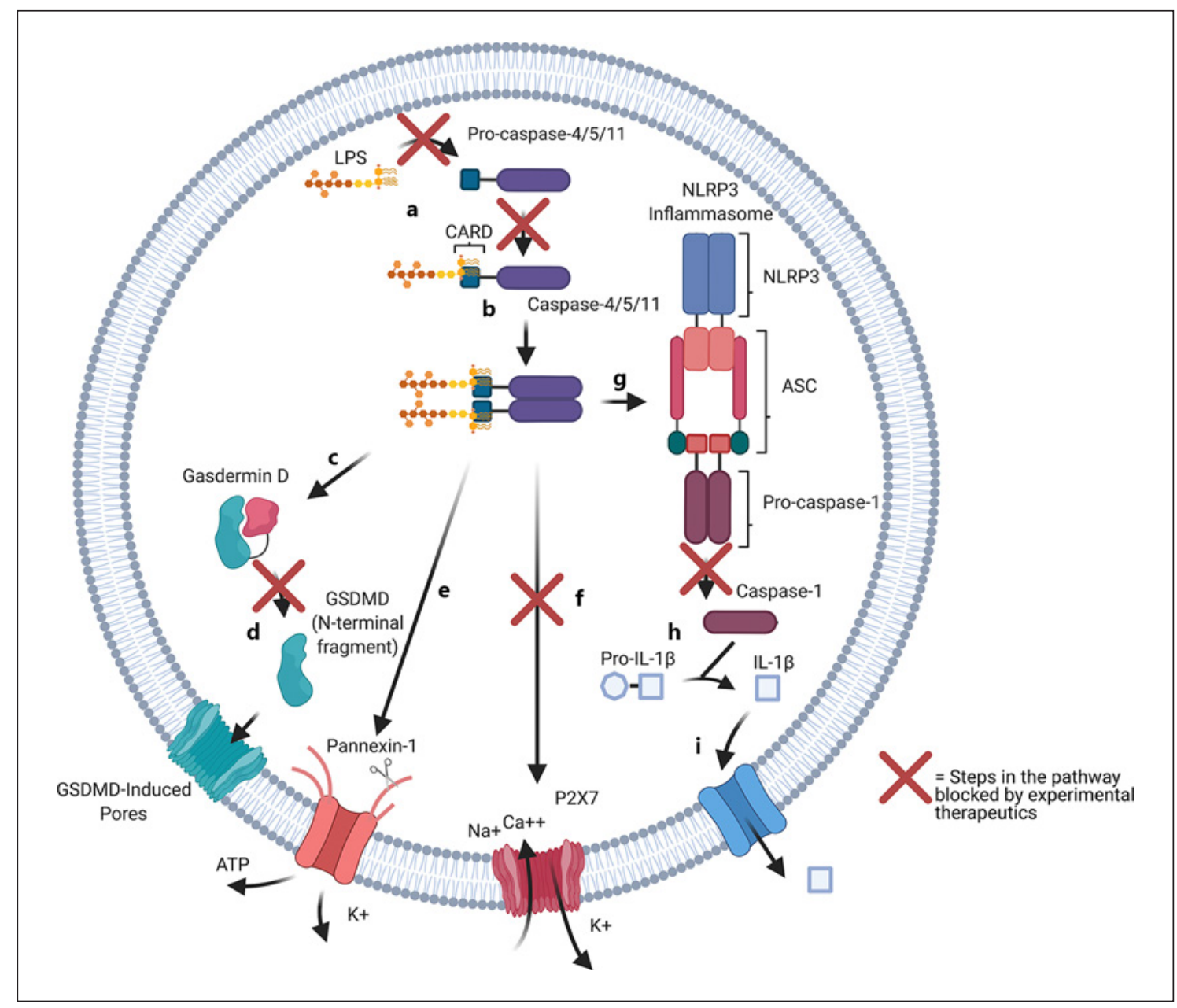

Fig. 2. Internal LPS signaling and pyroptosis pathways and potential therapies. a Cytoplasmic LPS binds to the CARD of procaspase-11/4/5. Pep19-2.5, an antiendotoxin peptide, can block LPS from binding to caspase-11/4/5, preventing further activation. b Activated caspase-11/4/5 begins oligomerizing and in turn activating the effectors of pyroptosis. For potential therapies, stearoyl LPC likely binds directly to caspase-11/4/5, blocking its activation and oligomerization. Azithromycin, a macrolide antibiotic, also likely interacts with caspase-11/4/5. However, its mechanism is less clear. c Caspase-11/4/5 cleaves GSDMD, generating N-terminal fragments. $\mathbf{d} \mathrm{N}$-terminal fragments of GSDMD localize to the cell membrane and oligomerize to form pores, promoting cell lysis. Necrosulfonamide directly binds to GSDMD, inhibiting its cleavage and subsequent oligomerization. e Caspase-11/4/5 cleaves Cterminal fragments of the membrane channel pannexin-1, inducing cytotoxic efflux of potassium and ATP. $\mathbf{f}$ Caspase-11/4/5 acti-

shock occur in TLR4-deficient mice, following priming of TLR3 with a ligand. Evidence suggests that either TLR3 or TLR4 priming is required for upregulation of caspase-11 expression, but subsequent cytotoxic events occur independent of TLR4 $[13,25]$. vates pannexin-1 and ATP efflux, which in turn activates P2X7 receptors, which begin forming pores. This results in potassium efflux, as well as sodium and calcium influx. Magnesium can inhibit the P2X7 receptor, likely by binding to extracellular ATP, which normally activates it. g Caspase-11/4/5 triggers the assembly of the NLRP3 inflammasome, which then cleaves procaspase- 1 into its active form. LL-37 is an antimicrobial cathelicidin which inhibits caspase- 1 activation and subsequent IL- $1 \beta$ secretion. $\mathbf{h}$ Caspase- 1 cleaves pro-IL- $1 \beta$ into IL- $1 \beta$. i IL- $1 \beta$ is secreted through membrane channels into surrounding tissue. Diagram created with Biorender.com. LPC, lysophosphatidylcholine; GSDMD, gasdermin D; LPS, lipopolysaccharide; CARD, caspase activation and recruitment domain; NLRP3, nucleotide-binding domain, leucine-rich-containing family, pyrin domain-containing-3.

\section{Caspases-11/4/5 Activate Effectors of Pyroptosis}

Caspase-11/4/5 oligomers trigger the assembly of the NLRP3 inflammasome. Once assembled, the NLRP3 inflammasome cleaves procaspase-1, converting it to its active form (shown in Fig. $2 \mathrm{~g}$ ). Caspase-1 then promotes the 
Table 1. Summary of effects of pyroptosis in sepsis

\begin{tabular}{ll}
\hline Effect of pyroptosis & Model \\
\hline $\begin{array}{l}\text { Increases local inflammation, IL-1 } \beta \text { and IL-18 production, induces } \\
\text { macrophage cell death }\end{array}$ & $\begin{array}{l}\text { Murine model with a single dose of } \\
\text { intraperitoneal LPS }\end{array}$ \\
\hline $\begin{array}{l}\text { Induces EC death, destruction of the lung endothelial barrier and } \\
\text { subsequent pulmonary edema, acute lung injury }\end{array}$ & $\begin{array}{l}\text { Murine model with a single dose of } \\
\text { intraperitoneal LPS }\end{array}$ \\
$\begin{array}{l}\text { Lytic death of renal tubular epithelial cells, abnormalities in renal } \\
\text { structure, acute kidney injury }\end{array}$ & $\begin{array}{l}\text { Murine model with a single dose of } \\
\text { intraperitoneal LPS }\end{array}$ \\
\hline
\end{tabular}

IL, interleukin; LPS, lipopolysaccharide; EC, endothelial cell.

cleavage of IL- $1 \beta$, and its secretion into surrounding tissue (shown in Fig. 2h, i). Notably, NLRP3 inhibitors prevent caspase- $4 / 5$-dependent IL- $1 \beta$ production by intracellular LPS [26].

Caspase-11 is also able to induce pyroptosis in caspase-1 knockout mice, independent of the NLRP3 inflammasome [27]. Caspase-11/4/5 oligomers are able to act directly on GSDMD, another effector of pyroptosis. Active caspase-11/4/5 cleaves GSDMD, generating Nand C-terminal fragments. These fragments, then, are able to localize to the cell membrane, oligomerize, and form membrane pores, resulting in cell lysis (shown in Fig. 2c, d) [28].

An additional proposed mechanism by which caspase-11 elicits cell death is via pannexin- 1 and purinergic receptor P2X7. Activated caspase-11 cleaves pannexin-1, a plasma membrane channel. Upon cleavage, large concentrations of ATP are released from the cell (shown in Fig. 2e). Released ATP is then able to act on the purinergic receptor P2X7, a cation channel, which then induces cytotoxic events including pore formation in the plasma membrane, potassium efflux, as well as sodium and calcium influx (shown in Fig. 2f). Extracellular LPS priming accentuates this pathway as it induces expression of caspase-11. It should also be noted that intracellular LPS appears to directly heighten the sensitivity of the P2X7 receptor to ATP release $[29,30]$.

In summary, caspases-11/4/5 is able to induce the secretion of IL- $1 \beta$ via NLRP3 and caspase- 1 activation, while also inducing cell lysis via both the perforin GSDMD and cation channel P2X7. It should also be noted that priming of cells by extracellular LPS upregulates expression of caspase-11/4/5, increasing activation and heightening the downstream effects of intracellular LPS pathways [14, 29].

Intracellular LPS Signaling

\section{Implications of LPS Internalization in Sepsis}

\section{Pyroptosis Increases Inflammation}

Pyroptosis of immune cells combats intracellular infection [31]. In vivo, this promotes clearance of intracellular pathogens as bacteria are released by pyroptosis into the extracellular space then phagocytosed by nearby macrophages or neutrophils [32].

During sepsis, as endotoxins enter the cytoplasm of macrophages, the cells undergo pyroptosis and increase local inflammation $[27,33,34]$. Studies have shown that caspase-1- and NLRP3-deficient mice undergo less macrophage pyroptosis, produce less IL- $1 \beta$, and are more resistant to LPS-induced septic shock [35-37]. Additionally, Wang et al. [20] demonstrated that inflammation was correlated with pyroptotic mononuclear cells in a prospective study of 60 trauma patients. They found a significant correlation between mononuclear cell pyroptosis and clinical measures including injury severity score, chronic health evaluation II score, and cytokine (IL-10, IL-18, and MCP-1) levels [20].

\section{Pyroptosis in the Endothelium Leads to Acute Lung Injury}

Sepsis often leads to destruction of the lung endothelial barrier, resulting in edema, hypoxemia, and death [38]. Damage to the lung endothelial barrier can be caused by caspase-11/4/5-mediated pyroptosis $[9,39,40]$. During sepsis, the death of ECs coincides with microtubule activation, actin cytoskeleton remodeling, and disassembly of endothelial adherens junctions. This endothelium dysfunction leads to cell retraction, gap formation, and subsequent edema. In vitro experiments have shown that intracellular LPS induces far greater cytotoxicity in ECs when than extracellular LPS $[9,41]$. However, priming of 
ECs by extracellular LPS upregulates caspases-4/5 [9]. ECs in the absence of priming can also undergo pyroptosis but required more prolonged exposure to intracellular LPS [9].

Destruction of the endothelial barrier results in protein-rich edema, activation of procoagulant pathways, release of pro-inflammatory cytokines, and influx of leukocytes. Deletion of caspase-11 in mice showed reduced endothelial permeability and enhanced survival upon LPS challenge [9]. Caspase-1 knockout mice also show a reduction of nearly $70 \%$ in EC death following sepsis [42]. This widespread lysis of ECs leads to acute lung injury in sepsis. Other than LPS internalization, Mitra et al. $[43,44]$ suggested that EC pyroptosis during sepsis is stimulated by the transport of microparticles containing activated caspase-1 and GSDMD from monocytes to ECs.

\section{Pyroptosis and Acute Kidney Injury}

It has also been reported that caspase-11-mediated pyroptosis occurs in renal tubular epithelial cells and may be a cause for acute kidney injury, another complication of sepsis [19, 45]. Ye et al. [19] examined acute kidney injury in a mouse model with caspase-11 knockout strains, along with accompanying in vitro work. The authors found that LPS injection resulted in lytic death of renal tubular epithelial cells, along with increased expression of caspase-11 and GSDMD in wild type strains. Caspase-11 knockout strains showed reduced abnormalities in renal structure and serum creatinine levels, indicating improved renal function [19]. Studies have found that oxidative stress and mitochondrial dysfunction also play a role in septic acute kidney injury [46, 47]. Li et al. [47] found that heme oxygenase protects mice against these damaging processes. Antioxidant compounds may also protect against these pathways. There is still a need for further research into pyroptosis in its induction of septic acute kidney injury, in order to find additional therapeutic targets. The effects of pyroptosis are summarized in Table 1.

\section{Past Therapeutic Efforts}

\section{LPS Neutralization Clinical Trials}

Monoclonal antibodies have been suggested for use in neutralizing circulating LPS and, thus, preventing its interaction with extracellular and intracellular receptors. However, antibody use has not been supported by clinical trials [7]. One tested antibody, HA-1A, proved to be ineffective in reducing a 14-day mortality in patients with sepsis or gram-negative endotoxemia [48]. HA-1A may have been affected by limited specific endotoxin-binding ability [49]. Ineffectiveness of LPS antibodies has been attributed to a number of factors including extreme sensitivity of humans to endotoxin when compared to animals and differences between preclinical and clinical experience [50].

Other forms of LPS antibodies may show more promise. These include antimicrobial peptides, short amino acid chains normally secreted by innate immune systems [51]. A recombinant $\mathrm{N}$-terminal fragment of bactericidal/ permeability-increasing protein (21) also known as Opebacan has shown efficacy in neutralizing LPS in vitro and murine models, following intravenous LPS infusion [52]. This drug has also shown efficacy in specific patient subgroups, such as in children with severe meningococcal sepsis [53].

\section{TLR4 and CD14 Antagonist Clinical Trials}

TLR4 antagonists have been largely unsuccessful as therapeutic agents in the treatment of sepsis. Eritoran seemed promising as a therapeutic treatment for sepsis during phase I and II clinical trials $[54,55]$. However, during phase III clinical trials, Eritoran was unable to significantly reduce mortality in patients with severe sepsis when compared to placebo [56].

TAK-242 is another small molecule developed to inhibit TLR4 signal transduction [57]. Evaluation of TAK242 in a murine model of endotoxin shock showed protection for mice from LPS-induced mortality [58]. During phase III clinical trials, the drug failed to reduce cytokine levels or decrease mortality rates in sepsis patients [59].

Monoclonal antibodies which antagonize CD14 have also been tested as therapy for sepsis. This includes IC14, a recombinant chimeric monoclonal antibody which binds to both membrane-bound and soluble forms of CD14. IC14 was well tolerated in a phase I clinical trial in sepsis patients [60]. Other anti-CD14 antibodies continue to be explored as a means of treatment [61].

\section{Novel Therapeutic Strategies}

\section{Caspase-11 Inhibitors}

To our knowledge, there have been no clinical trials with caspase inhibition therapy in sepsis patients. However, there have been promising results in mouse studies [62]. Li et al. [63] demonstrated that stearoyl lysophosphatidylcholine shows protective effects in vivo against 
sepsis in a mouse model using intraperitoneal injection of LPS. Lysophosphatidylcholine is a component of lipoproteins and appears to competitively inhibit caspase- 11 activation, GSDMD cleavage, and IL- $1 \beta$ release (shown in Fig. 2b). Additionally, azithromycin, a macrolide antibiotic, has been shown to inhibit LPS-induced IL-1 $\beta$ release in an LPS injection mouse sepsis model, by impairing caspase-11 activation (shown in Fig. 2b) [64]. Furthermore, Pep19-2.5 is an antiendotoxin peptide which protects against a sepsis model of endotoxemia in vivo and is able to impair caspase- 11 activation, IL- $1 \beta$ release, and pyroptotic cell death in human monocytes and macrophages in vitro (shown in Fig. 2a) [65].

\section{Other Caspase Inhibitors}

Caspase-1 and broad caspase inhibitors have also shown success in mouse models. LL-37, a human cathelicidin (antimicrobial peptide) is able to neutralize the action of intracellular LPS and inhibit pyroptosis, improving sepsis survival in mice. Its mechanisms of action include direct LPS binding and neutralization, as well as impairment of caspase-1 activation (shown in Fig. 2g) [66].

Studies have shown that broad caspase inhibitors can prevent lymphocyte apoptosis in cecal ligation and puncture murine models and improve survival outcomes [67, 68]. Weber et al. [68] administered VX-166, a broad caspase inhibitor which targets both apoptotic and inflammatory caspases, arresting lymphocyte apoptosis. Caspase- 1 has shown involvement in both monocyte pyroptosis and lymphocyte apoptosis [36]. Thus, blocking caspase- 1 could slow both of these pathological mechanisms. Another study using a broad caspase inhibitor found that it prevented endotoxemia-induced cardiac dysfunction in rats [69].

\section{GSDMD Inhibitors}

GSDMD may be inhibited by necrosulfonamide, a compound which prevents assembly of GSDMD oligomers and subsequent pore formation and pyroptotic death. This drug is able to improve survival from sepsis in mice following LPS administration (shown in Fig. 2d) [70]. Magnesium also appears to inhibit pyroptosis and GSDMD activation via inhibiting channel P2X7, preventing calcium influx into the cell upon LPS internalization (shown in Fig. 2f). As mentioned earlier, calcium influx is a contributor to pyroptotic cytotoxicity. Calcium influx also seems to be required for GSDMD pore formation [71].

Intracellular LPS Signaling

\section{Future Efforts}

Sepsis is a complex condition, requiring evolving strategies. TLR4 blocking and neutralization of circulating LPS seem like promising strategies, yet they have been largely unsuccessful $[48,72]$. Intracellular LPS signaling has certainly been neglected in the previous clinical trials as an important contributor to mortality in sepsis. Adjunctive therapy could simultaneously target both TLR4-independent and TLR4dependent LPS signaling pathways through a multipronged strategy including neutralization of LPS in circulation, blocking of TLR4 receptors, and blocking of internal LPS receptors. Intracellular LPS pathways have a number of targets including the inflammatory caspases-4/5/1 as well as their effectors, GSDMD, and the P2X7 receptor.

Current forms of sepsis treatment could show increased effectiveness when used adjunctively with internal LPS receptor antagonists. Similarly, previous forms of treatment which lacked efficacy, such as TLR4 blockers, may show added benefits as cointerventions with this new line of therapies.

There are numerous concerns involved with caspase therapy. Broad caspase inhibitors in large doses can have side effects including cytotoxicity. Caspases have also been shown to have other nonapoptotic functions, such as immune regulation. For example, caspase-1 also protects against colorectal tumorigenesis through secretion of IL-18, which appears to activate tumor suppressors such as IFN- $\gamma$ [73]. Disturbing these protective effects may produce adverse events in patients [74]. Finally, studies have shown that apoptosis can be initiated by only small amounts of activated caspase- 3 , meaning therapies might require a high degree of inhibition [75]. Selective caspases-4/5/1 inhibition could avoid some pitfalls of broad caspase inhibition. Ac-YVAD-cmk is a cell-permeable inhibitor of only the inflammatory caspases-1/4/5. An in vitro or animal study using Ac-YVAD-cmk could show promising results, given the documented roles of these caspases in sepsis [76].

\section{Conclusions}

Sepsis associated with gram-negative bacterial infection is affected through both extracellular and intracellular signaling. Intracellular LPS is able to bind to caspase- 11 in mice cells and caspase-4/5 in human cells, eliciting inflammation and pyroptotic cell death. A number of pathways for LPS internalization, including outer-membrane vesicles and delivery via HMGB1, have been discussed in the literature. Inside-out LPS signaling may explain the

Innate Immun 2021;13:323-332 
failure of LPS neutralization and TLR4 blockage in clinical trials due to the ongoing inflammatory effects of cytoplasmic LPS. In humans, intracellular signaling could be antagonized through targeting the inflammatory caspases-4/5/1 or their cellular effectors, including GSDMD and the P2X7 receptor. Conjunction therapy with LPS neutralization, TLR4 blocking, and inhibition of the human inflammatory caspases- $4 / 5 / 1$ presents an intriguing therapeutic option, which could be investigated in vivo. Ultimately, the evidence from animal models warrants clinical investigation into targeting of intracellular LPS in sepsis.

\section{Conflict of Interest Statement}

The authors have no conflicts of interest to declare.

\section{Funding Sources}

The study was supported by the Canadian Institutes of Health Research FDN143285 to Dr. Haibo Zhang and the National Nature Science Foundation of China 81270125 to Dr. Yimin Li.

\section{Author Contributions}

R.G. contributed to research and drafting of the manuscript. M.L. contributed to creation of the study outline, review of the literature, and drafting of the manuscript. P.Z. contributed to research and drafting of the manuscript. J.M. and Y.L. contributed to revision and editing of the manuscript. H.Z. contributed to design of the review outline, revision, editing, and writing of the manuscript.

\section{References}

1 Singer M, Deutschman CS, Seymour CW, Shankar-Hari M, Annane D, Bauer M, et al. The third international consensus definitions for sepsis and septic shock (Sepsis-3). JAMA. 2016;315(8):801-10.

2 Opal SM. Endotoxins and other sepsis triggers. Contrib Nephrol. 2010;167:14-24.

3 Poltorak A, He X, Smirnova I, Liu MY, Van Huffel C, Du X, et al. Defective LPS signaling in $\mathrm{C} 3 \mathrm{H} / \mathrm{HeJ}$ and $\mathrm{C} 57 \mathrm{BL} / 10 \mathrm{ScCr}$ mice: mutations in Tlr4 gene. Science. 1998;282(5396): 2085-8.

4 Yamamoto M, Akira S. Lipid A receptor TLR4-mediated signaling pathways. Adv Exp Med Biol. 2010;667:59-68.

5 Tisoncik JR, Korth MJ, Simmons CP, Farrar J, Martin TR, Katze MG. Into the eye of the cytokine storm. Microbiol Mol Biol Rev. 2012;76(1):16-32.

6 Cavaillon JM, Adib-Conquy M, Fitting C, Adrie $\mathrm{C}$, Payen D. Cytokine cascade in sepsis. Scand J Infect Dis. 2003;35(9):535-44.

7 Marshall JC. Why have clinical trials in sepsis failed? Trends Mol Med. 2014;20(4):195-203.

8 Lee JH, Del Sorbo L, Uhlig S, Porro GA, Whitehead T, Voglis S, et al. Intercellular adhesion molecule-1 mediates cellular crosstalk between parenchymal and immune cells after lipopolysaccharide neutralization. J Immunol. 2004;172(1):608-16.

9 Cheng KT, Xiong S, Ye Z, Hong Z, Di A, Tsang KM, et al. Caspase-11-mediated endothelial pyroptosis underlies endotoxemia-induced lung injury. J Clin Invest. 2017;127(11): 4124-35.

10 Cowan DB, Noria S, Stamm C, Garcia LM, Poutias DN, del Nido PJ, et al. Lipopolysaccharide internalization activates endotoxindependent signal transduction in cardiomyocytes. Circ Res. 2001;88(5):491-8.
11 Kopp F, Kupsch S, Schromm AB. Lipopolysaccharide-binding protein is bound and internalized by host cells and colocalizes with LPS in the cytoplasm: implications for a role of LBP in intracellular LPS-signaling. Biochim Biophys Acta. 2016;1863(4):660-72.

12 Shi J, Zhao Y, Wang Y, Gao W, Ding J, Li P, et al. Inflammatory caspases are innate immune receptors for intracellular LPS. Nature. 2014;514(7521):187-92.

13 Kayagaki N, Warming S, Lamkanfi M, Vande Walle L, Louie S, Dong J, et al. Non-canonical inflammasome activation targets caspase-11. Nature. 2011;479(7371):117-21.

14 Yang J, Zhao Y, Shao F. Non-canonical activation of inflammatory caspases by cytosolic LPS in innate immunity. Curr Opin Immunol. 2015;32:78-83.

15 Mazgaeen L, Gurung P. Recent advances in lipopolysaccharide recognition systems. Int J Mol Sci. 2020;21(2):379.

16 Vanaja SK, Russo AJ, Behl B, Banerjee I, Yankova M, Deshmukh SD, et al. Bacterial outer membrane vesicles mediate cytosolic localization of LPS and caspase-11 activation. Cell. 2016;165(5):1106-19.

17 Deng M, Tang Y, Li W, Wang X, Zhang R, Zhang X, et al. The endotoxin delivery protein HMGB1 mediates caspase-11-dependent lethality in sepsis. Immunity. 2018;49(4):74053.e7.

18 Viganò E, Diamond CE, Spreafico R, Balachander A, Sobota RM, Mortellaro A. Human caspase- 4 and caspase- 5 regulate the one-step non-canonical inflammasome activation in monocytes. Nat Commun. 2015;6: 8761.

19 Ye Z, Zhang L, Li R, Dong W, Liu S, Li Z, et al. Caspase-11 mediates pyroptosis of tubular epithelial cells and septic acute kidney injury. Kidney Blood Press Res. 2019:1-14.
20 Wang YC, Liu QX, Liu T, Xu XE, Gao W, Bai $\mathrm{XJ}$, et al. Caspase-1-dependent pyroptosis of peripheral blood mononuclear cells predicts the development of sepsis in severe trauma patients: a prospective observational study. Medicine. 2018;97(8):e9859.

21 Ellis TN, Kuehn MJ. Virulence and immunomodulatory roles of bacterial outer membrane vesicles. Microbiol Mol Biol Rev. 2010; 74(1):81-94.

22 Huebener P, Pradere JP, Hernandez C, Gwak GY, Caviglia JM, Mu X, et al. The HMGB1/ RAGE axis triggers neutrophil-mediated injury amplification following necrosis. J Clin Invest. 2015;125(2):539-50.

23 Shnyra A, Lindberg AA. Scavenger receptor pathway for lipopolysaccharide binding to Kupffer and endothelial liver cells in vitro. Infect Immun. 1995;63(3):865-73.

24 Hampton RY, Golenbock DT, Penman M, Krieger M, Raetz CR. Recognition and plasma clearance of endotoxin by scavenger receptors. Nature. 1991;352(6333):342-4.

25 Hagar JA, Powell DA, Aachoui Y, Ernst RK, Miao EA. Cytoplasmic LPS activates caspase-11: implications in TLR4-independent endotoxic shock. Science. 2013;341(6151): 1250-3.

26 Baker PJ, Boucher D, Bierschenk D, Tebartz C, Whitney PG, D'Silva DB, et al. NLRP3 inflammasome activation downstream of cytoplasmic LPS recognition by both caspase- 4 and caspase-5. Eur J Immunol. 2015;45(10): 2918-26.

27 Aachoui Y, Sagulenko V, Miao EA, Stacey KJ. Inflammasome-mediated pyroptotic and apoptotic cell death, and defense against infection. Curr Opin Microbiol. 2013;16(3): $319-26$. 
28 Yi YS. Caspase-11 non-canonical inflammasome: a critical sensor of intracellular lipopolysaccharide in macrophage-mediated inflammatory responses. Immunology. 2017; 152(2):207-17.

29 de Gassart A, Martinon F. Pyroptosis: caspase- 11 unlocks the gates of death. Immunity. 2015;43(5):835-7.

30 Yang D, He Y, Muñoz-Planillo R, Liu Q, Núñez G. Caspase-11 requires the pannexin-1 channel and the purinergic P2X7 pore to mediate pyroptosis and endotoxic shock. Immunity. 2015;43(5):923-32.

31 Aachoui Y, Leaf IA, Hagar JA, Fontana MF, Campos CG, Zak DE, et al. Caspase-11 protects against bacteria that escape the vacuole. Science. 2013;339(6122):975-8.

32 Miao EA, Rajan JV, Aderem A. Caspase-1-induced pyroptotic cell death. Immunol Rev. 2011;243(1):206-14.

33 Jorgensen I, Miao EA. Pyroptotic cell death defends against intracellular pathogens. Immunol Rev. 2015;265(1):130-42.

34 Rathinam VAK, Zhao Y, Shao F. Innate immunity to intracellular LPS. Nat Immunol. 2019;20(5):527-33.

35 Li P, Allen H, Banerjee S, Franklin S, Herzog L, Johnston C, et al. Mice deficient in IL-1 beta-converting enzyme are defective in production of mature IL-1 beta and resistant to endotoxic shock. Cell. 1995;80(3):401-11.

36 Sarkar A, Hall MW, Exline M, Hart J, Knatz N, Gatson NT, et al. Caspase-1 regulates Escherichia coli sepsis and splenic B cell apoptosis independently of interleukin-1beta and interleukin-18. Am J Respir Crit Care Med. 2006; 174(9):1003-10.

37 Lee S, Nakahira K, Dalli J, Siempos II, Norris PC, Colas RA, et al. NLRP3 inflammasome deficiency protects against microbial sepsis via increased lipoxin B4 synthesis. Am J Respir Crit Care Med. 2017;196(6):713-26.

38 Ware LB, Matthay MA. The acute respiratory distress syndrome. N Engl J Med. 2000; 342(18):1334-49.

39 Xi H, Zhang Y, Xu Y, Yang WY, Jiang X, Sha $\mathrm{X}$, et al. Caspase-1 inflammasome activation mediates homocysteine-induced pyropapoptosis in endothelial cells. Circ Res. 2016; 118(10):1525-39.

40 Yang J, Zhao Y, Zhang P, Li Y, Yang Y, Yang $Y$, et al. Hemorrhagic shock primes for lung vascular endothelial cell pyroptosis: role in pulmonary inflammation following LPS. Cell Death Dis. 2016;7(9):e2363.

41 Singla S, Machado RF. Death of the endothelium in sepsis: understanding the crime scene. Am J Respir Cell Mol Biol. 2018;59(1):3-4.

42 Lai D, Tang J, Chen L, Fan EK, Scott MJ, Li Y, et al. Group 2 innate lymphoid cells protect lung endothelial cells from pyroptosis in sepsis. Cell Death Dis. 2018;9(3):369.

43 Mitra S, Exline M, Habyarimana F, Gavrilin MA, Baker PJ, Masters SL, et al. Microparticulate caspase 1 regulates gasdermin $\mathrm{d}$ and pulmonary vascular endothelial cell injury. Am J Respir Cell Mol Biol. 2018;59(1):56-64.
44 Mitra S, Wewers MD, Sarkar A. Mononuclear phagocyte-derived microparticulate caspase- 1 induces pulmonary vascular endothelial cell injury. PLoS One. 2015;10(12): e0145607.

45 Gómez H, Kellum JA. Sepsis-induced acute kidney injury. Curr Opin Crit Care. 2016 Dec; 22(6):546-53

46 Guo LP, Liu SX, Yang Q, Liu HY, Xu LL, Hao $\mathrm{YH}$, et al. Erratum to "effect of thymoquinone on acute kidney injury induced by sepsis in BALB/c mice". Biomed Res Int. 2020;2020: 3182919.

47 Li HB, Zhang XZ, Sun Y, Zhou Q, Song JN, $\mathrm{Hu} \mathrm{ZF}$, et al. HO-1/PINK1 regulated mitochondrial fusion/fission to inhibit pyroptosis and attenuate septic acute kidney injury. Biomed Res Int. 2020;2020:2148706.

48 McCloskey RV, Straube RC, Sanders C, Smith SM, Smith CR. Treatment of septic shock with human monoclonal antibody HA-1A. A randomized, double-blind, placebo-controlled trial. CHESS Trial Study Group. Ann Intern Med. 1994;121(1):1-5.

49 Stephens R, Mythen M, editors. Update on anti-endotoxin therapies. Intensive care medicine. New York, NY: Springer; 2003.

50 Hurley JC. Towards clinical applications of anti-endotoxin antibodies; a re-appraisal of the disconnect. Toxins. 2013;5(12):2589-620.

51 Divyashree M, Mani MK, Reddy D, Kumavath $\mathrm{R}$, Ghosh $\mathrm{P}$, Azevedo V, et al. Clinical applications of antimicrobial peptides (AMPs): where do we stand now? Protein Pept Lett. 2020;27(2):120-34.

52 Lin Y, Kohn FR, Kung AH, Ammons WS Protective effect of a recombinant fragment of bactericidal/permeability increasing protein against carbohydrate dyshomeostasis and tumor necrosis factor-alpha elevation in rat endotoxemia. Biochem Pharmacol. 1994;47(9): 1553-9.

53 Levin M, Quint PA, Goldstein B, Barton P, Bradley JS, Shemie SD, et al. Recombinant bactericidal/permeability-increasing protein (rBPI21) as adjunctive treatment for children with severe meningococcal sepsis: a randomised trial. rBPI21 Meningococcal Sepsis Study Group. Lancet. 2000;356(9234):961-7.

54 Lynn M, Rossignol DP, Wheeler JL, Kao RJ, Perdomo CA, Noveck R, et al. Blocking of responses to endotoxin by E5564 in healthy volunteers with experimental endotoxemia. J Infect Dis. 2003;187(4):631-9.

55 Tidswell M, Tillis W, Larosa SP, Lynn M, Wittek AE, Kao R, et al. Phase 2 trial of eritoran tetrasodium (E5564), a toll-like receptor 4 antagonist, in patients with severe sepsis. Crit Care Med. 2010;38(1):72-83.

56 Opal SM, Laterre PF, Francois B, LaRosa SP, Angus DC, Mira JP, et al. Effect of eritoran, an antagonist of MD2-TLR4, on mortality in patients with severe sepsis: the ACCESS randomized trial. JAMA. 2013;309(11):1154-62.
57 Takashima K, Matsunaga N, Yoshimatsu M, Hazeki K, Kaisho T, Uekata M, et al. Analysis of binding site for the novel small-molecule TLR4 signal transduction inhibitor TAK-242 and its therapeutic effect on mouse sepsis model. Br J Pharmacol. 2009;157(7):1250-62.

58 Sha T, Sunamoto M, Kitazaki T, Sato J, Ii M, Iizawa Y. Therapeutic effects of TAK-242, a novel selective Toll-like receptor 4 signal transduction inhibitor, in mouse endotoxin shock model. Eur J Pharmacol. 2007;571(23):231-9.

59 Rice TW, Wheeler AP, Bernard GR, Vincent JL, Angus DC, Aikawa N, et al. A randomized, double-blind, placebo-controlled trial of TAK-242 for the treatment of severe sepsis. Crit Care Med. 2010;38(8):1685-94.

60 Reinhart K, Glück T, Ligtenberg J, Tschaikowsky K, Bruining A, Bakker J, et al. CD14 receptor occupancy in severe sepsis: results of a phase I clinical trial with a recombinant chimeric CD14 monoclonal antibody (IC14). Crit Care Med. 2004;32(5):1100-8.

61 Polat G, Ugan RA, Cadirci E, Halici Z. Sepsis and septic shock: current treatment strategies and new approaches. Eurasian J Med. 2017; 49(1):53-8.

62 Pfalzgraff A, Weindl G. Intracellular lipopolysaccharide sensing as a potential therapeutic target for sepsis. Trends Pharmacol Sci. 2019;40(3):187-97.

63 Li W, Zhang W, Deng M, Loughran P, Tang $\mathrm{Y}$, Liao $\mathrm{H}$, et al. Stearoyl lysophosphatidylcholine inhibits endotoxin-induced caspase-11 activation. Shock. 2018;50(3):33945.

64 Gualdoni GA, Lingscheid T, Schmetterer KG, Hennig A, Steinberger P, Zlabinger GJ. Azithromycin inhibits IL-1 secretion and non-canonical inflammasome activation. Sci Rep. 2015;5:12016.

65 Pfalzgraff A, Heinbockel L, Su Q, Brandenburg K, Weindl G. Synthetic anti-endotoxin peptides inhibit cytoplasmic LPS-mediated responses. Biochem Pharmacol. 2017;140: 64-72.

$66 \mathrm{Hu} \mathrm{Z}$, Murakami T, Suzuki K, Tamura H, Reich J, Kuwahara-Arai K, et al. Antimicrobial cathelicidin peptide LL-37 inhibits the pyroptosis of macrophages and improves the survival of polybacterial septic mice. Int Immunol. 2016;28(5):245-53.

67 Hotchkiss RS, Tinsley KW, Swanson PE, Chang KC, Cobb JP, Buchman TG, et al. Prevention of lymphocyte cell death in sepsis improves survival in mice. Proc Natl Acad Sci U S A. 1999;96(25):14541-6.

68 Weber P, Wang P, Maddens S, Wang PSh, Wu R, Miksa M, et al. VX-166: a novel potent small molecule caspase inhibitor as a potential therapy for sepsis. Crit Care. 2009;13(5): R146.

69 Nevière R, Fauvel H, Chopin C, Formstecher P, Marchetti P. Caspase inhibition prevents cardiac dysfunction and heart apoptosis in a rat model of sepsis. Am J Respir Crit Care Med. 2001;163(1):218-25. 
70 Rathkey JK, Zhao J, Liu Z, Chen Y, Yang J, Kondolf HC, et al. Chemical disruption of the pyroptotic pore-forming protein gasdermin $\mathrm{D}$ inhibits inflammatory cell death and sepsis. Sci Immunol. 2018;3(26):eaat2738.

71 Wang D, Zheng J, Hu Q, Zhao C, Chen Q, Shi $\mathrm{P}$, et al. Magnesium protects against sepsis by blocking gasdermin $\mathrm{D} \mathrm{N}$-terminal-induced pyroptosis. Cell Death Differ. 2020 Feb;27(2): 466-81.
72 Angus DC, Birmingham MC, Balk RA, Scannon PJ, Collins D, Kruse JA, et al. E5 murine monoclonal antiendotoxin antibody in gramnegative sepsis: a randomized controlled trial. E5 Study Investigators. JAMA. 2000;283(13): 1723-30.

73 Zaki MH, Vogel P, Body-Malapel M, Lamkanfi M, Kanneganti TD. IL-18 production downstream of the Nlrp3 inflammasome confers protection against colorectal tumor formation. J Immunol. 2010;185(8):4912-20.

74 Aziz M, Jacob A, Wang P. Revisiting caspases in sepsis. Cell Death Dis. 2014;5:e1526.
75 Méthot N, Huang J, Coulombe N, Vaillancourt JP, Rasper D, Tam J, et al. Differential efficacy of caspase inhibitors on apoptosis markers during sepsis in rats and implication for fractional inhibition requirements for therapeutics. I Exp Med. 2004;199(2):199_ 207.

76 Garcia-Calvo M, Peterson EP, Leiting B, Ruel R, Nicholson DW, Thornberry NA. Inhibition of human caspases by peptide-based and macromolecular inhibitors. J Biol Chem. 1998;273(49):32608-13. 